\title{
THE APPLICATION OF FLUKA TO DOSIMETRY AND RADIATION THERAPY
}

\author{
V. Andersen ${ }^{1}$, F. Ballarini ${ }^{2,3}$, G. Battistoni ${ }^{3}$, F. Cerutti ${ }^{3,4}$, A. Empl ${ }^{1}$, A. Fassò ${ }^{5,6}$, A. Ferrari ${ }^{3,5}$, \\ M. V. Garzelli ${ }^{1,4}$, A. Ottolenghi ${ }^{2,3}$, H. Paretzke ${ }^{7}$, L. Pinsky ${ }^{1 *}$, J. Ranft ${ }^{5,8}$, P. Sala ${ }^{9}$, T. Wilson $^{10}$ and M. Zankl $^{7}$ \\ ${ }^{1}$ University of Houston, Houston, TX, USA \\ ${ }^{2}$ University of Pavia, Italy \\ ${ }^{3}$ INFN, Italy \\ ${ }^{4}$ University of Milan, Italy \\ ${ }^{5}$ CERN, Geneva, Switzerland \\ ${ }^{6}$ SLAC, Stanford, CA, USA \\ ${ }^{7}$ GSF, Neuherberg, Germany \\ ${ }^{8}$ University of Leipzig, Germany \\ ${ }^{9}$ ETH, Zurich, Switzerland \\ ${ }^{10}$ NASA-JSC, Houston, TX, USA
}

\begin{abstract}
The FLUKA Monte Carlo code has been evolving over the last several decades and is now widely used for radiation shielding calculations. In order to facilitate the use of FLUKA in dosimetry and therapy applications, supporting software has been developed to allow the direct conversion of the output files from standard CT-scans directly into a voxel geometry for transport within FLUKA. Since the CT-scan information essentially contains only the electron density information over the scanned volume, one needs the specific compositions for each voxel individually. We present here the results of a simple algorithm to assign tissues in the human body to one of four categories: soft-tissue, hard-bone, trabecular-bone and porouslung. In addition, we explore the problem of the pathlength distributions in porous media such as trabecular bone. A mechanism will be implemented within FLUKA to allow for variable multipal fixed density materials to accommodate the pathlength distributions discovered.
\end{abstract}

\section{INTRODUCTION}

Monte Carlo transport codes like FLUKA are useful for many purposes, and one of those is the simulation of the effects of radiation traversing the human body. In particular, radiation has been used in cancer therapy for a long time, and recently this has been extended to include heavy ion particle beams. This capability is also of interest to NASA because of the nature of the radiation environment in space $^{(1)}$. When in space, the crew members' bodies are continually traversed by virtually all forms of radiation. In assessing the risk that this exposure causes, heavy ions are of primary importance. These arise from the external space radiation itself, as well as the fragments that result from interactions during the traversal of that radiation through any intervening material, including the body tissue. Thus, the capability to characterise the details of the radiation field accurately within a human body that is subjected to such external 'beams' is of critical importance.

In order to provide this capability, making use of the widest practical application of known physics, the FLUKA Monte Carlo code has been extended over the last several years to include the ability to

*Corresponding author: pinsky@uh.edu simulate heavy-ion interactions more completely ${ }^{(2)}$. Currently, FLUKA is available with internal event generators that are capable of simulating inelastic nuclear reactions down to an incident lab kinetic energy of $\sim 100 \mathrm{MeV} \mathrm{A}^{-1(3)}$. Work on extending the internal event generator's capability all the way down to the reaction thresholds, as well on updating and improving the existing capabilities on a continuing basis is in progress, as new data become available.

One common hurdle in the use of all Monte Carlo radiation transport codes is the difficulty of providing the detailed geometry information about the system to be modelled. This is particularly problematic when the object is as complex as the human body. The difficulty is multiplied by the requirement not only of an accurate positional geometric description, but also the absolute need to attach to that positional information the details of the composition and density of the actual material. In cases where the human body is the object of the simulation, the technique of using information from CT-scans is common, especially when the nature of the incident beam is restricted to electromagnetic radiation and electrons. This is because the primary information from a CT-scan is a measure of the local distribution of electron density in the object scanned. Raw CTscan files are generally represented as scan layers of 
fixed thicknesses with data from individual cells within each sequential layer being given in raster pixel format. The three-dimensional volume element made up of each of these pixels in a given layer is referred to as a 'voxel'.

In order to be able to accept these raw CT-scan inputs, FLUKA has been modified to allow the direct embedding of a region composed of these internal voxels within any normal FLUKA geometric description. Therefore, for example, one could embed a CT-scan-based voxel human phantom region within a spacecraft that was otherwise described using the normal FLUKA capabilities. Similarly, a CT-scan-based voxel phantom can be embedded in the external laboratory environment geometry description for simulation of acceleratorbased exposures.

Unfortunately, by itself, a raw CT-scan is not as useful in providing a basis for the transport geometry when hadronic beams are applied due to the general lack of direct compositional information. This can be remedied if there is some external process whereby the composition can be associated with each individual voxel. In some cases, such as the soft tissue in the human body, the use of a common global generic composition is a very good approximation. However, when dealing with the whole body or large fractions of it, this technique produces incorrect results because some organs, particularly bone and the lungs, have both notably different compositions and porous structures.

\section{ASSIGNING COMPOSITION}

In order to address these issues, we have explored the development of an algorithm to distinguish bone and lung tissue from the other soft-tissues within the body. The additional small problem of the existence of gas pockets, for example in the stomach and bowel, is easily included owing to the very dramatic difference in density.

Our intention is to provide a compositional tag for each voxel that is either a generic 'soft-tissue', that is global for all soft-tissue, or one of the special tissue types including hard-bone, trabecular-bone and porous-lung. It should be emphasised that the actual measured densities will be used after being scaled to the generic composition that has been assigned based on the X-ray cross sections, which are, typically, proportional to the electron density. For example, the density of each soft-tissue voxel can still vary, even though the presumed elemental composition remains the same.

The challenges for bone and lung tissue are actually of two distinct kinds. First, there is the initial problem of simply distinguishing this tissue from the surrounding soft tissue. This is generally a pattern recognition problem, which yields to well-known techniques that take advantage of density variations. Lung tissue is relatively easy to distinguish, but bone is more problematic. The thin 'hard-bone' shell that surrounds most human bone tissue is filled with an inner trabecular-bone tissue that is a combination of hard-bone filaments and a variety of different kinds of marrow. This composite trabecular-bone tissue is typically unresolved on many large-scale CT-scans, and has an average density that could match that of the surrounding soft tissue. However, the general property that it is internal to the hard-bone shell, typically provides a handle on the problem of distinguishing it from the external soft-tissue. We have developed several software tools to facilitate the development of these algorithms.

In general, the work reported here has been done using two separate sources for CT-scans. The first is a whole-body image of an adult male ${ }^{(2)}$ that has been previously analysed by hand to assign, voxel-byvoxel, the organ membership along with the corresponding material composition. The second image is that of trabecular-bone tissue and represents a $1.2 \mathrm{~cm}$ cubic volume entirely with the region of trabecular bone with a voxel size of $40 \mu \mathrm{m}$. In this case, all voxels are identified either as entirely hard-bone or entirely interstitial marrow ${ }^{(4)}$.

Figure 1 shows the displays employed to develop the appropriate algorithms. Figure 1a contains the whole-body image where the voxel densities of the slices shown have been displayed as a corresponding linear grey scale. Figure $1 \mathrm{~b}$ shows the same image processed by a simple density-based algorithm in order to distinguish hard-bone from soft-tissue. Note that there is a histogram tool towards the top that displays the actual density profile for the vertical cross-hair-line and a fit to that profile by the algorithm. The results are displayed for comparison in the image itself. One can see that the hard-bone is easily distinguished from the soft-tissue in a general sense. The major difficulties are with the boundary voxels and distinguishing the internal trabecularbone from general soft-tissue.

The boundary between hard-bone and soft-tissue can be problematic because the actual physical boundary between the outer hard-bone surface and the surrounding soft-tissue generally occurs in the midst of a voxel rather than close to or along a voxel side. The current algorithm employs the simplest reasonable solution and assigns the voxel either as hard-bone or soft-tissue. A more complex solution that may be employed is similar to the one that has been developed to deal with the trabecular-bone regions as discussed in the next section.

Figure 1b also shows that the lungs are easily distinguished from the surrounding tissue, and even the central region where the major pulmonary arteries and veins enter the lungs, the distinctions 
(a)

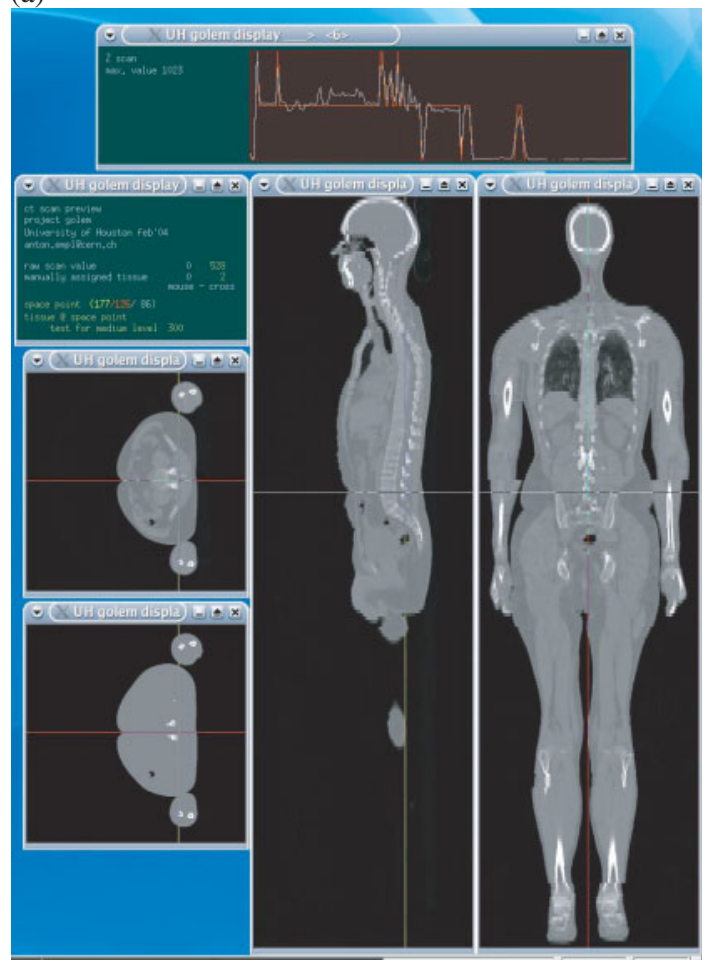

(b)

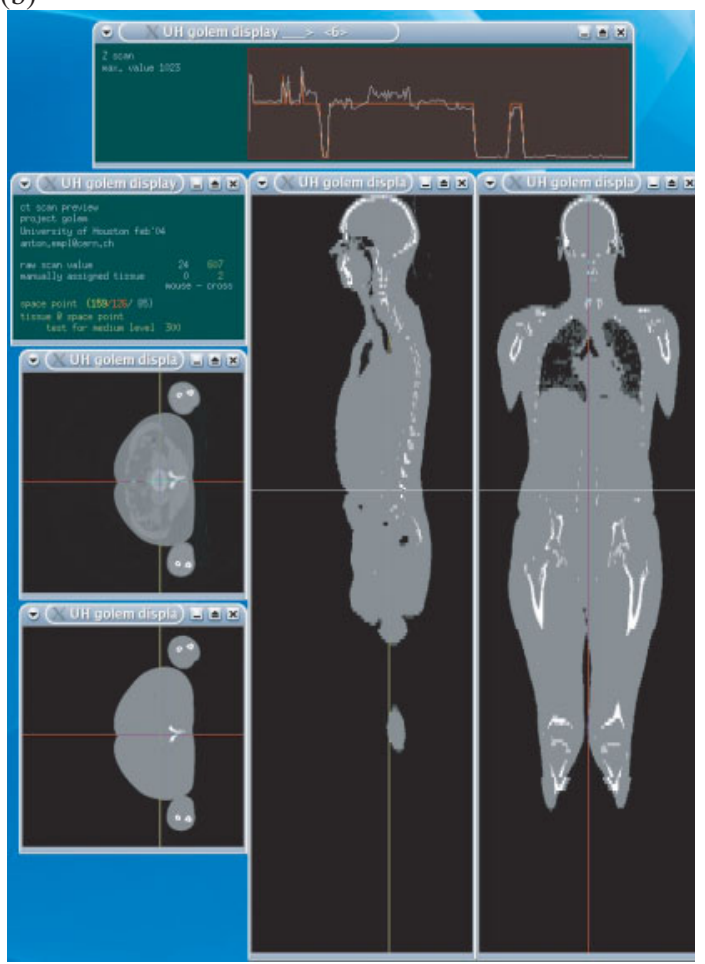

Figure 1. Screen shots are presented of the software tool developed to assess the performance of the tissue assignment algorithm. (a) A slice from the 'Golem' CT-scan, where the raw density values are displayed on a linear brightness scale as pixels. (b) The result of filtering this image with the simple threshold algorithm. Note the histogram tool that displays the raw data across the slice and the values from the algorithm superimposed.

are fairly clear. The lung boundaries present the same challenge as does the bone boundary, and are amenable to the same solutions. These simple algorithms are subject to improvement, but given the level of success that has already been achieved by our methods, considerable progress has been made in getting the material properties into the Monte Carlo geometry inputs.

\section{TRABECULAR-BONE POROSITY}

The porosity of trabecular-bone and the lungs provides a separate distinct challenge. The problem stems from the fact that the details of porosity occur at a scale that is not practical to evaluate in large volume whole-body CT-scans. As such, if the voxel is treated as if it possessed some mean density with a homogeneous composition, then all tracks traversing the voxel with the same trajectorylength in the voxel will see the identical material ?pathlength in $\mathrm{g} \mathrm{cm}^{-2}$. In reality, the pseudo-random porous nature of the material will actually give rise to a distribution of pathlengths. Further, in the case of trabecular-bone, this distribution can also have a directional dependence.

Several FLUKA runs were performed on the $40 \mu \mathrm{m}$ resolution trabecular-bone CT-scan image using rays to determine the actual pathlengths within the hard-bone component of the trabecular-bone. This particular example of trabecular-bone possessed $\sim 10 \%$ of its voxels as hard-bone with the remainder being treated as vacuum for our purposes here. Hard-bone is taken to have a density of $1.920 \mathrm{gm} \mathrm{cm}^{-2}$ and the pathlength distributions are all given in terms of $\mathrm{g} \mathrm{cm}^{-2}$ of hard-bone traversed per centimetre of total pathlength traversed. The data are limited to rays with a minimum $0.5 \mathrm{~cm}$ pathlength in the trabecular-bone volume.

Figure 2a shows the pathlength distribution for $\sim 100,000$ rays which are incident isotropically on the CT-scan volume. It is seen that there are essentially no rays that manage to avoid all hard-bone voxels, and the mean is $\sim 0.1 \mathrm{gm} \mathrm{cm}^{-2}$ per $\mathrm{cm}$. Figure $2 \mathrm{~b}$ is an expanded view of the long pathlength tail of the same distribution.

Figure 3a shows the result for another 100,000 rays, but for which the direction is strictly vertical, 
V. ANDERSEN ET AL.

(a) Path length distribution for isotropic rays in trabecular-bone

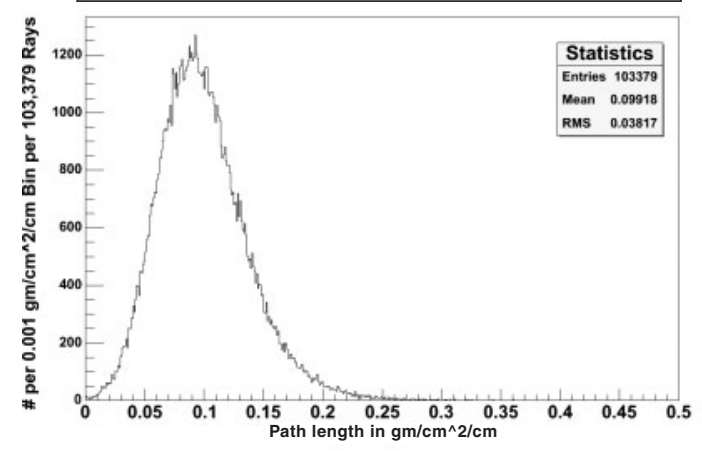

(b) Long path length distribution tail for isotropic rays in trabecular-bone

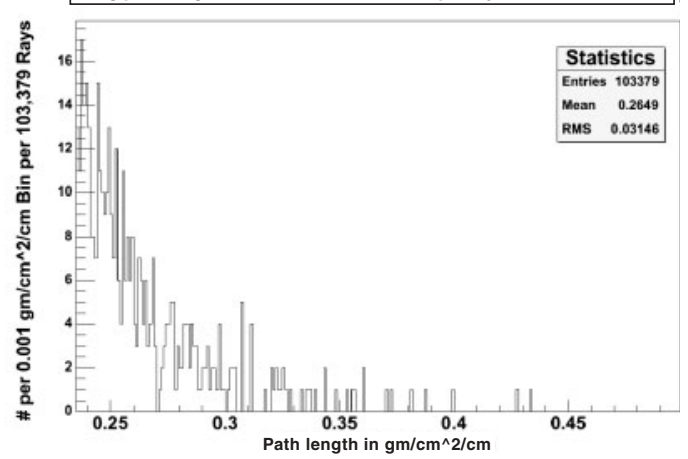

Figure 2. (a) This graph displays the pathlength distributions in trabecular-bone for an isotropic irradiation. All pathlengths included in the plot have a minimum $0.5 \mathrm{~cm}$ absolute length, and the results are plotted as $\mathrm{g} \mathrm{cm}^{-2}$ of hardbone per actual centimetre of pathlength. (b) An enlargement of the long pathlength tail. The distribution fits a normal distribution quite well.
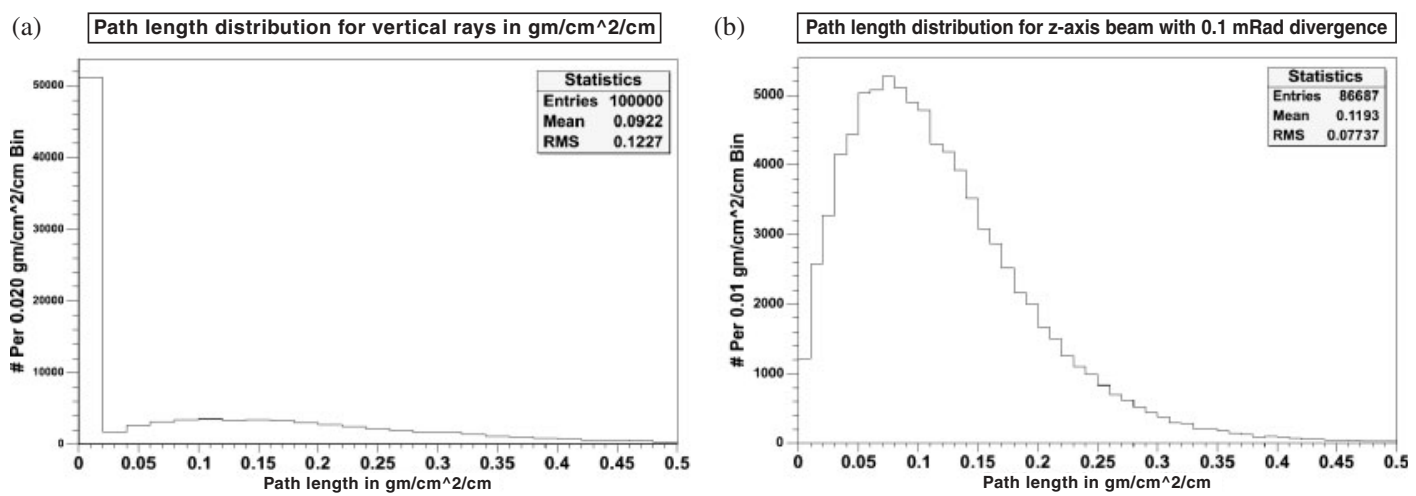

Figure 3. This graph displays the pathlength distributions similar to those of Figure 1, but for tracks incident along the longitudinal axis of the bone (the $z$-axis) in our coordinate system. (a) A plot for rays strictly parallel to the $z$-axis, shows the surprising behaviour of possessing a considerable peak at zero pathlength. (b) A plot for rays diverging from the $z$-axis with this divergence spread uniformly over angle up to $100 \mathrm{mrad}$, and the large zero pathlength peak is entirely missing.

along the axis of the bone. Slightly more than $50 \%$ of these rays pass through the full $1.2 \mathrm{~cm}$ of trabecularbone without striking any hard-bone voxels! This implies that strictly along the axis of the bone the pathlength variation is a very important feature for particle transport. Figure 3 a shows a similar set of rays, but where the angle with respect to the bone axis is distributed uniformly over a $100 \mathrm{mrad}$ cone. However, note that even this modest angular deviation from the strict longitudinal axis causes a rapid reduction of this anomaly with the result tending towards the isotropic distribution with the virtual disappearance of the zero pathlength rays.

Figure 4 shows the result for a lateral beam (perpendicular to the bone axis) with no angular spread. A comparison with Figure $2 \mathrm{a}$ shows that the lateral pathlength distribution is comparable to the general isotropic distribution.
Thus, it appears as if a strategy can be deployed wherein the pathlength used is compensated by a normal distribution fit to the isotropic pathlength distribution, with the provision that for particle directions that are very close to axial along the bone, a second distribution should be used that is a combination of (a) 50\% no hard-bone and (b) $50 \%$ of twice the standard isotropic pathlength distribution.

To implement this strategy, rather than vary the actual pathlength, the mechanism to be employed within FLUKA will be to provide for a new kind of material that has a variable material type. Then when a particle enters such a medium the program will select the material and density to be used for that particle randomly from a fixed number of choices based on a distribution function. This will allow the effect of the porosity of the medium to be properly 


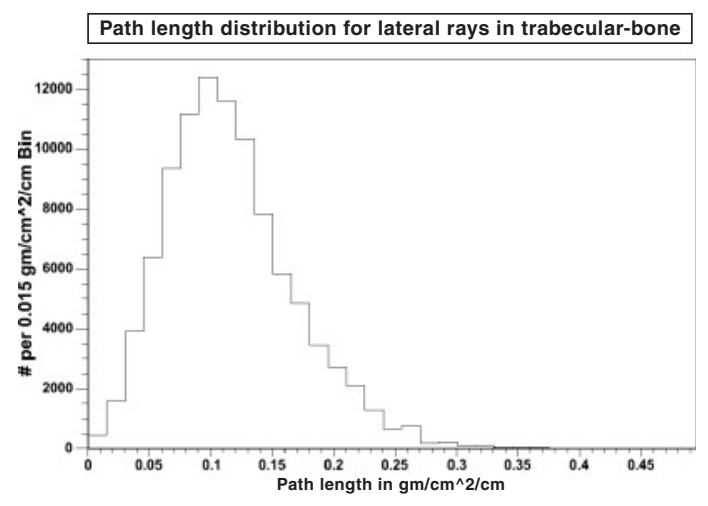

Figure 4. This graph plot shows the pathlength distribution that results from an exposure to rays that are exactly parallel to one of the axes that is perpendicular (the reference to $x$-axis here is intended to be a generic axis perpendicular to the longitudinal axis of the bone). Like Figure $3 \mathrm{a}$, there is no divergence in the beam, but unlike that figure, there is no corresponding zero pathlength peak.

simulated and the net effect on a traversing beam of charged particles will be to spread out the energy loss effects as well as the other interactions probabilities.

\section{ACKNOWLEDGEMENTS}

We would like to acknowledge the use of the socalled 'Golem' whole-body CT-scan dataset along with the version that resulted from the efforts of Laura De Biaggi in her detailed voxel-by-voxel assignment of individual organs to that dataset and the similar efforts of Maria Zankl. We are also grateful to Professor Gemunu Gunaratne of the University of Houston for providing access to the trabecular-bone CT-scan employed, as well as for his efforts in producing graphic images of the CT-scan. Finally, we gratefully acknowledge Professor Michael A. K. Liebschner of the Bioengineering Department of Rice University for permission to use the trabecular-bone CT-scan, which Professor Gunaratne had obtained from him originally. This work was partially supported by the EC (contract no. FI6R-CT-2003-508842, 'RISC-RAD'), NASA (contracts NAG8-1901 and NAG8-1658) and the Institute for Space Systems Operations at the University of Houston.

\section{REFERENCES}

1. Andersen, V. et al. The FLUKA code for space applications: recent developments. Adv. Space Res. (in press) (2004).

2. Ballarini, F. et al. Role of shielding in modulating the effects of Solar Particle Events: Monte Carlo calculation of physical and "biological" dose in different organs. Adv. Space Res. (in press) (2004).

3. Fassò, A. et al. The physics models of FLUKA: status and recent developments CHEP-2003-MOMT005, June 2003. p. 10. Talk given at 2003 Conference for Computing in High-Energy and Nuclear Physics (CHEP'03), La Jolla, CA, 24-28 March 2003. Published in eConf C0303241:MOMT005, 2003, e-Print Archive: hep-ph/0306267 (2003).

4. Gunaratne, G. and Leibschner, M. Private communication (2004). 